\title{
Co-Administration of Risperidone and Sodium Valproate Causing Bilateral Chronic Exertional Lower Leg Compartment Syndrome
}

\author{
Ravi K. Ray', Muhammad A. Bashir ${ }^{1}$, Sudhir Rao ${ }^{2}$ \\ ${ }^{1}$ Guy's \& St Thomas' NHS Foundation Trust, London, UK \\ ${ }^{2}$ Princess Royal University Hospital NHS Trust, Farnborough, Kent, UK \\ Email: raviray@hotmail.com
}

Received 14 February 2014; revised 18 March 2014; accepted 27 March 2014

Copyright (C) 2014 by authors and Scientific Research Publishing Inc.

This work is licensed under the Creative Commons Attribution International License (CC BY). http://creativecommons.org/licenses/by/4.0/

(c) (i) Open Access

\begin{abstract}
We present the case of a 24-year-old man, who was a keen runner, developing chronic bilateral lower leg compartment syndrome following co-administration of Risperidone and Sodium Valproate. Having had failed medical and physical therapies, he underwent surgical decompression of his more problematic left lower leg compartments. In the interval between sequential fasciotomies, control of his psychiatric symptoms allowed the dose of his Risperidone to be reduced, resulting in a greater exercise tolerance. Ultimately he no longer required the contra-lateral lower leg compartments to be decompressed surgically. He had returned to his original level of activity with no further psychotic episodes.
\end{abstract}

Keywords

Risperidone, Compartment, Syndrome

\section{Introduction}

All drugs exhibit side effects and when these are associated with acute surgical emergencies, doctors and nurses in specialties other than surgery and orthopaedics need to maintain an awareness of these. Primary care physicians can be alert to chronic conditions but emergency doctors and nurses should realize that through knowledge and experience of the medical fraternity, conditions like compartment syndrome are less likely to be neglected. We attempt to highlight this with our case report on chronic exertional compartment syndrome with the co-ad- 
ministration of two anti-psychotic drugs, but other case reports manage the same with more acute scenarios.

\section{Case Report}

Our patient was a fit young man who was a keen runner. He developed an acute psychosis at the age of 24 years and, following initial psychiatric assessment, which was started on Olanzapine. This was ineffective in controlling his psychosis and was therefore changed to the atypical antipsychotic Risperidone and the anti-epileptic drug Sodium Valproate, which controlled his psychiatric symptoms very well. After the initiation of Risperidone (4 mg daily) and Sodium Valproate (500 mg daily), he noticed symptoms of pain and cramping in both lower legs which came on only after exercise and the diagnosis of chronic bilateral lower leg exertional compartment syndrome was made.

He altered his exercise regimen but could not effect a significant change in his symptoms. He then saw his general practitioner who made a referral to the Orthopaedic department at Queen Mary's Hospital, Sidcup. He was seen and assessed by the consultant at that time and referred to physiotherapy. No amount of therapy reduced his symptoms and he therefore re-attended clinic requesting further treatment. The senior author made the clinical diagnosis of chronic exertional compartment syndrome then undertook decompression of his left lower leg deep and superficial posterior compartments. Under tourniquet, the operation was performed as a day case under a general anaesthetic releasing all four compartments through fasciotomies of the left leg. This went uneventfully without damage to adjacent structures.

Initially asked to rest both legs during the post-operative period, the patient then attempted to return to sport. His psychosis was very well controlled with the co-administration of Risperidone and Sodium Valproate, so his psychiatrist recommended a reduction of Risperidone from $4 \mathrm{mg}$ to $2 \mathrm{mg}$. Several months later, when a re-assessment was made, a further halving of this dose took place, satisfactorily titrating his medical treatment to his psychiatric symptoms.

When reviewed in clinic by the Orthopaedic department several months later, it was noted that the surgical scars from the left leg compartment decompressions had healed well with the leg lacking any signs of injury to nerves or vessels.

On further questioning, in preparation for right lower leg compartment decompressions, it appeared that with each reduction in dose of Risperidone, the patient was able to exercise longer and faster on the treadmill, achieving almost the same level of activity as his pre-morbid state at the last consultation. The dose of Sodium Valproate remained unchanged throughout this entire period and only occasional regular analgesics were prescribed in addition to the anti-psychotics in the early post-operative phase.

The patient therefore declined any further physiotherapy, medical treatment or surgical decompression and was promptly discharged from the clinic. Advice was given about recurrence if the dose was increased and the British National Formulary was advised about the case through the "Yellow Card" route of suspected adverse drug reactions in 2009 when the case occurred.

\section{Discussion}

It seems clear that with initiation of Risperidone, this patient developed chronic bilateral lower leg exertional compartment syndrome, which then abated on reduction in the dose of Risperidone.

The co-administration of Sodium Valproate seems inconsequential, as there appears to be a dose related response to Risperidone. One cannot rule out the fact that co-administration of Risperidone and other drugs may well be causing compartment syndrome as the other relevant literature often shows multiple drug therapy in these patients.

Previous reports through the United States Food \& Drug Administration (FDA) [1] have noted injectable depot forms of Risperidone in the form of Risperdal Consta ${ }^{\circledR}$ to have caused compartment syndrome in $0.05 \%$ (26 of 52,838) of users but the data is unclear about any further details. Data from the same source suggests all occurred within one month of initial administration. Claims from the same website suggest only 2 reports that have been made of compartment syndrome in patients taking generic Risperidone though the source itself suggests data which has been input by patients and may be unreliable.

A study [2] has suggested that rhabdomyolysis and compartment syndrome occurred with coadministration of risperidone and simvastatin. There are also reports of simvastatin causing myositis and subsequent compartment syndrome warranting acute fasciotomies [3] [4], thereby implying that it may be statins as well as Risperidone 
as the cause for these compartment syndromes.

In 2009, the British National Formulary [5] did not record compartment syndrome as even a rare side effect of Risperidone, though myalgia and arthralgia are mentioned within the more common side effects section. Since we have sent them a Yellow Card, and as evidence is accrued in the literature, the BNF has included rhabdomyolysis as a rare side effect now.

\section{References}

[1] Could Risperidone Cause Compartment Syndrome. eHealthMe.com [Internet]. Cited 13 February 2014. http://www.ehealthme.com/ds/risperidone/compartment+syndrome

[2] Webber, M.A., Mahmud, W., Lightfoot, J.D. and Shekhar, A. (2004) Rhabdomyolysis and Compartment Syndrome with Coadministration of Risperidone and Simvastatin. Journal of Psychopharmacology, 18, 432-434.

[3] Ramdass, M.J., Singh, G. and Andrews, B. (2007) Simvastatin-Induced Bilateral Leg Compartment Syndrome and Myonecrosis Associated with Hypothyroidism. Postgraduate Medicine Journal, 83, 152-153.

[4] Walker, J.L., Smith, G.H., Gaston, M.S. and Robinson, C.M. (2008) Spontaneous Compartment Syndrome in Association with Simvastatin-Induced Myositis. Emergency Medicine Journal, 25, 305-306.

[5] Committee, J.F. (2013) British National Formulary (BNF) 66. Pharmaceutical Press, London, 1128. 